\title{
The fish fauna of the Jacaré-Guaçu River basin, Upper Paraná River basin
}

\author{
André Luiz Henríques Esguícero ${ }^{1,2}$ \& Marlene Sofia Arcifa ${ }^{1}$ \\ ${ }^{1}$ Programa de Pós-graduação em Biologia Comparada, Laboratório de Limnologia, \\ Departamento de Biologia, Universidade de São Paulo - USP, \\ Av. Bandeirantes, n. 3900, CEP 14040-901, Ribeirão Preto, SP, Brazil \\ ${ }^{2}$ Corresponding author: André Luiz. Henríques Esguícero, e-mail: andre.esguicero@gmail.com
}

ESGUÍCERO, A.L.H. \& ARCIFA, M.S. The fish fauna of the Jacaré-Guaçu River basin, Upper Paraná River basin. Biota Neotrop. 11(1): http://www.biotaneotropica.org.br/v11n1/en/abstract?article+bn01811012011.

\begin{abstract}
This study aimed to evaluate the fish fauna composition and distribution in different environments, up- and downstream the Gavião Peixoto Dam, in the Jacaré-Guaçu River basin. Most of the sampled ichthyofauna is autochthonous, and the majority of the species belongs to the orders Characiformes and Siluriformes. The order Characiformes comprised most of the specimens caught, Astyanax altiparanae being the most abundant species. All the studied environments were characterized by the massive occurrence of accidental species and low similarity between their ichthyofauna. In general, a greater number of species and higher values of the diversity index were observed downstream of the dam. The fish diversity in the studied habitats was positively correlated with the water body depth, and negatively correlated with the water conductivity. This last correlation may be an indicative of the negative influence of the pollution of the Jacaré-Guaçu basin on the local fish fauna.

Keywords: Neotropical fishes, stream ecology, communities' structure, freshwater fishes, environmental degradation.
\end{abstract}

ESGUÍCERO, A.L.H. \& ARCIFA, M.S. Ictiofauna da bacia do Rio Jacaré-Guaçu, bacia do Alto Rio Paraná. Biota Neotrop. 11(1): http://www.biotaneotropica.org.br/v11n1/pt/abstract?article+bn01811012011.

Resumo: Este estudo teve como objetivo avaliar a composição e distribuição da ictiofauna em diferentes ambientes, a montante e a jusante da PCH Gavião Peixoto, na bacia do Rio Jacaré-Guaçu. A grande maioria da ictiofauna amostrada é autóctone e pertencente às ordens Characiformes e Siluriformes. A ordem Characiformes foi a que apresentou o maior número de espécimes capturados, sendo Astyanax altiparanae a espécie mais abundante em toda a bacia. Todos os ambientes estudados foram caracterizados pela alta ocorrência de espécies acidentais e apresentaram baixa similaridade entre suas ictiofaunas, principalmente quando comparados os ambientes a jusante com os ambientes a montante do barramento da PCH Gavião Peixoto. No geral, os ambientes a jusante apresentaram maior número de espécies e maiores valores do índice de diversidade. A diversidade de peixes nos ambientes estudados foi positivamente correlacionada com a profundidade do corpo d'água e negativamente correlacionada com a condutividade da água. Esta última correlação pode ser um indicativo da influência negativa da poluição registrada na bacia do Rio Jacaré-Guaçu sobre a ictiofauna local.

Palavras-chave: peixes Neotropicais, ecologia de rios, estrutura de comunidade, peixes de água doce, degradação ambiental. 


\section{Introduction}

The Jacaré-Guaçu River, focus of this study, is one of the main tributaries of the right margin of the Tietê River, one of the largest tributaries of the Upper Paraná River Basin. The Upper Paraná River comprises all the Paraná River drainage upstream the Salto de Sete Quedas, a natural geographic barrier submerged by the formation of the Itaipu reservoir in 1982 (Agostinho \& Julio Jr. 2002). Denominated as a natural ichthyofaunistic province (Géry 1969), this basin has been considered by many authors as a region with elevated endemism rate (Vari 1988, Langeani 1989, Castro et al. 2003). The estimated ichthyofauna of the Upper Paraná River basin is 310 species, distributed in 38 families and 11 orders (Langeani et al. 2007). The Siluriformes and Characiformes are the richest orders, comprising the majority of the dominant groups in the lotic environments. Despite the high importance and representativeness of the Upper Paraná River basin for the Neotropical fish diversity, a depletion of its fish fauna has been progressing in the last two decades (Agostinho et al. 2007). Several factors may be responsible for this event: the presence of a high number of dams; introductions of alien species; overfishing; and the drainage of large urban and agricultural areas (Agostinho \& Julio Jr. 1999).

The principal focus of this study is an ichthyofaunistic inventory of the Jacaré Guaçu-River, with the analysis of the composition and abundance of the fish fauna in different habitats. Besides, some comments about the influence of anthropic impacts over the fish fauna composition are made.

\section{Materials and Methods}

\section{Study area}

Jacaré-Guaçu (Figure 1) is a fourth-order river with a catchment area of approximately $4,108 \mathrm{~km}^{2}$. Its basin comprises 34 counties, totalizing 498,172 inhabitants (IBGE, 2000 census, www.ibge.com.br). The main river receives a large contribution of domestic and industrial sewage, mainly through its tributaries. The river is characterized by high concentrations of fecal coliforms (4000 NMP.100 mL ${ }^{-1}$, CETESB, www.cetesb.sp.gov.br), pesticides (Corbi et al. 2006) and low percentage (8\%) of domestic sewage removal in its basin (www.sigrh.sp.gov.br). The natural vegetation of this basin is impacted by anthropic actions, mainly farming and cattle ranching (CATI/Lupa 2008, www.cati.sp.gov.br/projetolupa). The remnants of natural vegetation are mainly composed of seasonal semideciduous and riparian forests and cerrado (savannas) (Hueck \& Seibert 1981). The region has a tropical climate, with at least three months of dry season, and the annual mean temperature ranges from 18 to $20{ }^{\circ} \mathrm{C}$ (Nimer 1989). The river belongs to the Upper Paraná River basin, and flows into the Ibitinga Reservoir (Tietê River), the headwaters being located in the Serra de Itaqueri (town of São Carlos, São Paulo State, Brazil).

In a previous study, Albino (1987) recorded 77 fish species, distributed in 19 families, in Jacaré-Guaçu River. This author highlights the absence of several large-sized migratory fish species, such as Salminus brasiliensis (Cuvier, 1816), Pseudoplatystoma fasciatum (Linnaeus, 1766), and Zungaro jahu (Ihering, 1898). The dams in the Jacaré-Guaçu River were considered the main responsible for the reduction of the reproductive conditions, dispersion and survival of the local fish fauna.

There are three dams on the Jacaré-Guaçu River: Gavião Peixoto Dam, closed in 1913, and Lobo and Santana dams which were closed in 1936 and 1964, respectively. Only Gavião Peixoto Dam has a fish passage, which after evaluations in the summers of 2007/2008,
2008/2009, and 2009/2010 (Esguícero \& Arcifa 2010a, b) proved to be inefficient for upstream migration. Taking into account the diversity and complex migratory behavior of South American fishes, most fish ladders are inefficient for downstream migration (Agostinho et al. 2007), and probably the fish ladder of Gavião Peixoto Dam is no exception.

\section{Studied habitats}

The Jacaré-Guaçu River, a tributary and a floodplain lake were studied upstream and downstream the Gavião Peixoto Dam (Figure 1), in addition to its reservoir. Each habitat was surveyed aiming to characterize the species of macrophytes, margins and their vegetation, the substrate type of the bottom, and the width of the area (Table 1). Moreover, some physical and chemical factors were recorded (Table 1): average depth, measured at a fixed point; current velocity, which was estimated by the method of a floating object (Brower \& Zar 1984); vertical water transparency, estimated with a $30 \mathrm{~cm}$-white Secchi disk; and temperature, dissolved oxygen, electrical conductivity, and $\mathrm{pH}$, measured in the superficial layer of the water with Yellow Springs Inc. equipment.

\section{Sampling strategy}

A total of 15 sampling campaigns were conducted. Samplings were carried out during the dry-season (June), from 2007 to 2009 , and during the rainy-season, once a month from November through February, from 2007 to 2010 . The sampling gear employed consisted of 10-m-long (used in the Jacaré-Guaçu River) or 5-m-long (used in the tributaries) gill nets with $1.5,4.0$, and $6.0 \mathrm{~cm}$ mesh, between adjacent knots, and a sieve $(1 \times 0.70 \mathrm{~m})$ with a $0.5 \mathrm{~mm}$ mesh. The effort was standardized and similar at each station. The gill nets were placed at sunset, and set during ca. 15 hours in the river sections, and 20 passes of the rectangular sieve were made, in each sampling period. The specimens were fixed in $10 \%$ formalin immediately after their capture, and transferred to $70 \%$ ethanol. Voucher specimens (7539 and 7554 to 7627) were deposited at the Laboratório de Ictiologia de Ribeirão Preto (LIRP) of the Departamento de Biologia of the Faculdade de Filosofia, Ciências e Letras de Ribeirão Preto, Universidade de São Paulo, Brazil.

\section{Data analysis}

In addition to the species abundance in the entire sampled region, the species frequency and constancy (C) were calculated in each sampling station. The species dominance, assumed as the proportion of a given species in the total sample, and constancy (C) were calculated in each sampling station. The species were classified as: constant $(\mathrm{C}>50 \%)$, accessory $(50 \% \geq \mathrm{C} \geq 25 \%)$, accidental $(\mathrm{C}<25 \%)$, and absent $(\mathrm{C}=0 \%)$. The species were identified according to its origin: autochthonous - native from the Upper Paraná River basin; allochthonous - native from other Neotropical basin; and exotic - native from another continent.

The fish fauna similarity between the studied habitats was evaluated with the Jaccard index (J'). For a better visualization of the similarities, a dendrogram was obtained, with the UPGMA cluster analysis of Jaccard index, using the software NTSYS-pc 2.02. The Shannon-Wiener index (H') was employed to analyze the fish diversity in the habitats, and the values were compared with the Student's $t$-test (Ludwig and Reynolds 1988). The influences of physical and chemical factors on the fish diversity were evaluated with the Pearson's correlation analysis, using the H' and the average values of the physical and chemical factors of each habitat. All the statistical analyses were performed using the software Past 1.90 (Hammer et al. 2001). 
Fish fauna of the Jacaré-Guaçu River basin

\begin{tabular}{|c|c|c|c|c|}
\hline \multirow[t]{8}{*}{ South America } & $\begin{array}{l}\text { Sampling } \\
\text { codes }\end{array}$ & Sampling sites & $\begin{array}{l}\text { Altitude } \\
\text { (m) }\end{array}$ & $\begin{array}{l}\text { Geographical } \\
\text { coordinates }\end{array}$ \\
\hline & s01 & Jacaré-Guaçu River downstream & 419 & $\begin{array}{l}21^{\circ} 52^{\prime} 00^{\prime \prime} \mathrm{S} \\
48^{\circ} 31^{\prime} 28^{\prime \prime} \mathrm{W}\end{array}$ \\
\hline & S02 & Floodplain Lake downstream & 419 & $\begin{array}{l}21^{\circ} 52^{\prime} 02^{\circ} \mathrm{S} \\
48^{\circ} 31^{\prime} 22^{\prime \prime} \mathrm{W}\end{array}$ \\
\hline & $\mathrm{S} 03$ & Boa Esperança River & 421 & $\begin{array}{l}21^{\circ} 52^{\prime} 16^{\prime \prime} \mathrm{S} \\
48^{\circ} 31^{\prime} 02^{\prime \prime} \mathrm{W}\end{array}$ \\
\hline & S04 & Dam Reservoir & 433 & $\begin{array}{l}21^{\circ} 50^{\prime} 46^{\prime \prime} S \\
48^{\circ} 29^{\prime} 22^{\prime \prime} W\end{array}$ \\
\hline & S05 & Jacaré-Guaçu River upstream & 475 & $\begin{array}{l}21^{\circ} 51^{\prime} 59^{\prime \prime} \mathrm{S} \\
48^{\circ} 16^{\prime} 42^{\prime \prime} \mathrm{W}\end{array}$ \\
\hline & S06 & Floodplain Lake upstream & 481 & $\begin{array}{l}21^{\circ} 51^{\prime} 53^{\prime \prime} \mathrm{S} \\
48^{\circ} 19^{\prime} 42^{\prime \prime} \mathrm{W}\end{array}$ \\
\hline & S07 & Chibarro River & 490 & $\begin{array}{l}21^{\circ} 51^{\prime} 52^{\prime \prime} \mathrm{S} \\
48^{\circ} 16^{\prime} 09^{\prime \prime} \mathrm{W}\end{array}$ \\
\hline
\end{tabular}

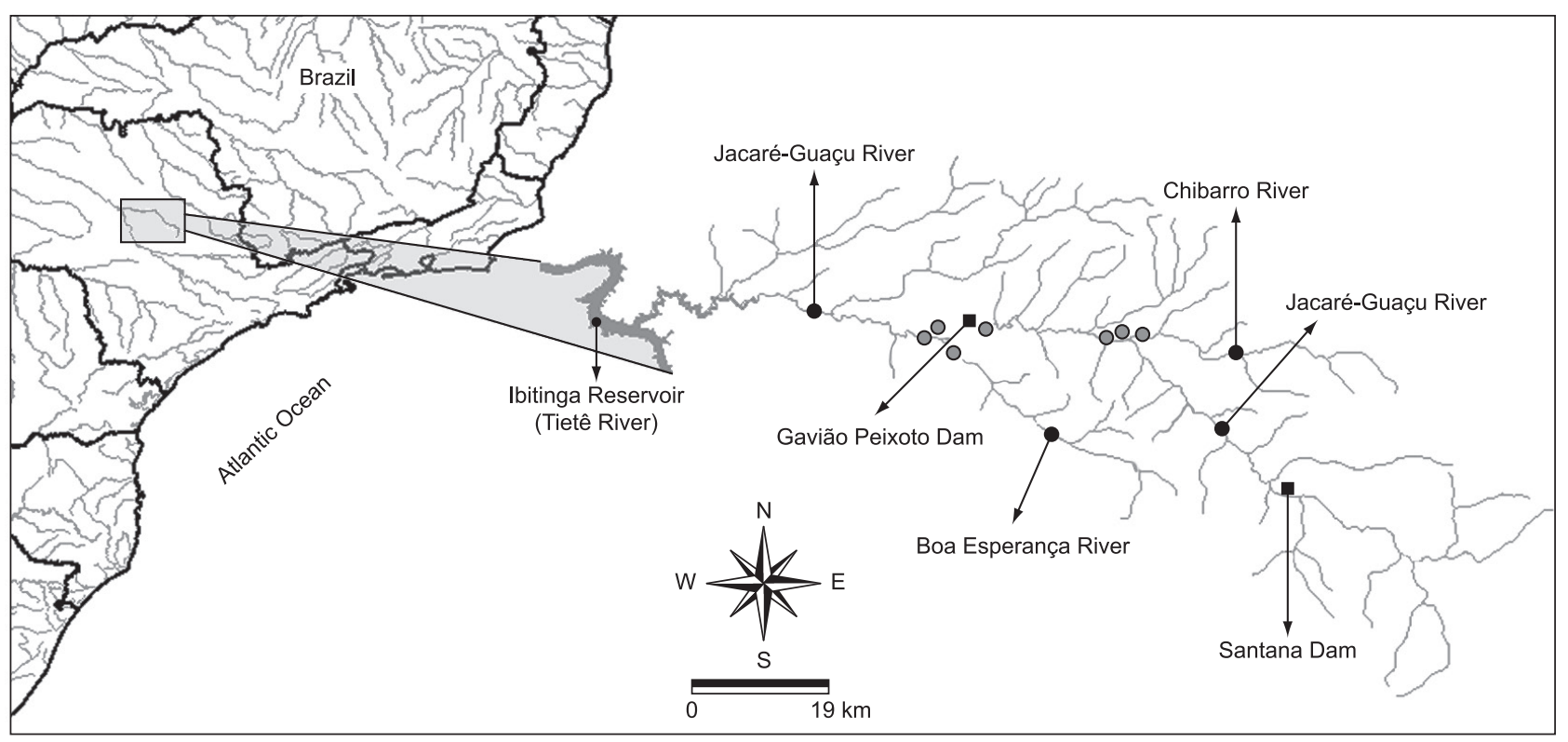

Figure 1. Location of the sampling stations (S) in the Jacaré-Guaçu River basin.

\section{Results}

A total of 6,844 specimens, belonging to six orders, 24 families, 59 genera, and 82 species (Table 2), were collected in the seven studied habitats (Figure 1). Approximately $86.3 \%$ of the captured specimens belonged to the order Characiformes, $9 \%$ to the order Siluriformes, $2.6 \%$ to the order Gymnotiformes, $1.3 \%$ to the order Perciformes, $0.7 \%$ to the order Cyprinodontiformes, and $0.2 \%$ to the order Synbranchiformes. Of the 24 sampled families, nine belonged to the order Characiformes, $70.9 \%$ of the specimens of this order included in the family Characidae (Table 2 ). The order Characiformes also showed the greatest number of species (53.7\%). Among the other species, $32.9 \%$ belonged to the order Siluriformes, $6.1 \%$ to the order Perciformes, $3.7 \%$ to the order Gymnotiformes, $2.4 \%$ to the order Cyprinodontiformes, and $1.2 \%$ to the order Synbranchiformes. The Characiformes Astyanax altiparanae, Steindachnerina insculpta, and A. fasciatus together comprised $50 \%$ of all specimens collected (Table 2), being the most abundant species of the entire studied region. These species were also dominant in at least more than half of the study sites. Four other species were dominant, but in only one of the sites. Of the 82 sampled species, 11 occurred in all the seven sites, and just $A$. altiparanae was identified as constant in all of them.

The majority of the collected species $(78 \%)$ was native from the Upper Paraná River basin (Table 2), and only four of them were known as migratory species, based on Carolsfeld et al. (2003). Alien species corresponded to ca. $16 \%$ of the species, most of them collected only downstream of the Gavião Peixoto Dam.

A higher number of fish species was captured downstream the dam (72 species downstream and 49 upstream the dam). All the habitats were characterized by a high occurrence of accidental species (Table 2). Besides, there was a low similarity among the fish fauna of these habitats (J'<58\%) (Table 3), the Jacaré-Guaçu River downstream the dam (S01) and the Boa Esperança River (S03) being the most similar sites. The dendrogram (Figure 2) highlights two groups, one composed of habitats located upstream the dam and the other of habitats located downstream the dam. 
Table 1. Characteristics of the studied habitats (S; Figure 1) in the Jacaré-Guaçu River Basin. Minimum and values of physical and chemical factors in each studied habitat, from 2007 to 2010. Mean value and standard deviation in parentheses.

\begin{tabular}{|c|c|c|c|c|c|c|c|}
\hline Habitats & S01 & S02 & S03 & S04 & S05 & S06 & S07 \\
\hline Depth (m) & $\begin{array}{c}1.2-2.9 \\
(1.9 \pm 1.1)\end{array}$ & $\begin{array}{c}0.4-1.0 \\
(0.8 \pm 0.3)\end{array}$ & $\begin{array}{c}0.6-2.2 \\
(1.3 \pm 0.9)\end{array}$ & $\begin{array}{c}1.4-1.8 \\
(1.7 \pm 0.2)\end{array}$ & $\begin{array}{c}0.9-2.4 \\
(1.6 \pm 0.8)\end{array}$ & $\begin{array}{c}0.5-1.6 \\
(1.0 \pm 0.7)\end{array}$ & $\begin{array}{c}0.8-1.9 \\
(1.3 \pm 0.6)\end{array}$ \\
\hline $\begin{array}{l}\text { Current } \\
\text { velocity }(\mathrm{m} / \mathrm{s})\end{array}$ & $\begin{array}{c}0.3-0.6 \\
(0.5 \pm 0.2)\end{array}$ & $0.0-0.0$ & $\begin{array}{c}0.4-0.7 \\
(0.5 \pm 0.2)\end{array}$ & $\begin{array}{c}0.1-0.3 \\
(0.2 \pm 0.2)\end{array}$ & $\begin{array}{c}0.2-0.5 \\
(0.4 \pm 0.2)\end{array}$ & $0.0-0.0$ & $\begin{array}{c}0.4-0.7 \\
(0.6 \pm 0.2)\end{array}$ \\
\hline $\begin{array}{l}\text { Vertical } \\
\text { transparency } \\
\text { (m) }\end{array}$ & $\begin{array}{c}0.4-0.6 \\
(0.5 \pm 0.1)\end{array}$ & $\begin{array}{c}0.2-0.8 \\
(0.6 \pm 0.3)\end{array}$ & $\begin{array}{c}0.5-1.1 \\
(0.8 \pm 0.4)\end{array}$ & $\begin{array}{c}0.3-0.7 \\
(0.5 \pm 0.3)\end{array}$ & $\begin{array}{c}0.3-0.5 \\
(0.4 \pm 0.2)\end{array}$ & $\begin{array}{c}0.4-0.6 \\
(0.5 \pm 0.2)\end{array}$ & $\begin{array}{c}0.3-0.5 \\
(0.4 \pm 0.1)\end{array}$ \\
\hline $\begin{array}{l}\text { Water } \\
\text { temperature } \\
\left({ }^{\circ} \mathrm{C}\right)\end{array}$ & $\begin{array}{c}18.3-26.6 \\
(23.7 \pm 6.9)\end{array}$ & $\begin{array}{c}14.9-29.7 \\
(24.2 \pm 7.5)\end{array}$ & $\begin{array}{c}17.9-25.5 \\
(22.9 \pm 6.8)\end{array}$ & $\begin{array}{c}17.8-26.7 \\
(23.4 \pm 6.1)\end{array}$ & $\begin{array}{c}17.2-26.0 \\
(24.1 \pm 7.3)\end{array}$ & $\begin{array}{c}14.1-27.4 \\
(24.4 \pm 7.2)\end{array}$ & $\begin{array}{c}18.2-25.1 \\
(24.2 \pm 6.2)\end{array}$ \\
\hline $\mathrm{pH}$ & $\begin{array}{c}6.0-6.7 \\
(6.4 \pm 0.2)\end{array}$ & $\begin{array}{c}5.4-6.5 \\
(6.3 \pm 0.3)\end{array}$ & $\begin{array}{c}6.5-6.8 \\
(6.7 \pm 0.1)\end{array}$ & $\begin{array}{c}5.8-6.9 \\
(6.7 \pm 0.2)\end{array}$ & $\begin{array}{c}5.9-6.5 \\
(6.3 \pm 0.2)\end{array}$ & $\begin{array}{c}6.0-6.8 \\
(6.7 \pm 0.2)\end{array}$ & $\begin{array}{c}6.2-7.1 \\
(6.8 \pm 0.3)\end{array}$ \\
\hline $\begin{array}{l}\text { Dissolved } \\
\text { oxygen } \\
\left(\mathrm{mg} \cdot \mathrm{L}^{-1}\right)\end{array}$ & $\begin{array}{c}6.4-7.9 \\
(7.7 \pm 0.1)\end{array}$ & $\begin{array}{c}2.7-7.5 \\
(7.4 \pm 0.2)\end{array}$ & $\begin{array}{c}5.5-7.9 \\
(7.8 \pm 0.2)\end{array}$ & $\begin{array}{c}5.9-7.9 \\
(7.7 \pm 0.4)\end{array}$ & $\begin{array}{c}5.2-8.3 \\
(7.8 \pm 0.5)\end{array}$ & $\begin{array}{c}4.8-7.8 \\
(7.5 \pm 0.4)\end{array}$ & $\begin{array}{c}3.4-6.6 \\
(5.4 \pm 1.1)\end{array}$ \\
\hline $\begin{array}{l}\text { Water } \\
\text { conductivity } \\
\left(\mu \mathrm{S} . \mathrm{cm}^{-1}\right)\end{array}$ & $\begin{array}{c}57.1-69.0 \\
(64.6 \pm 4.1)\end{array}$ & $\begin{array}{c}46.5-121.1 \\
(74.2 \pm 52.2)\end{array}$ & $\begin{array}{c}36.2-64.2 \\
(59.2 \pm 12,3)\end{array}$ & $\begin{array}{c}56.6-72.8 \\
(65.4 \pm 9.9)\end{array}$ & $\begin{array}{c}58.7-68.7 \\
(65.2 \pm 6.2)\end{array}$ & $\begin{array}{c}58.2-69.5 \\
(66.6 \pm 5.9)\end{array}$ & $\begin{array}{c}59.7-83.2 \\
(71.2 \pm 14.4)\end{array}$ \\
\hline Macrophytes & Brachiaria spp. & $\begin{array}{l}\text { Salvinia auriculata } \\
\text { Salvinia biloba } \\
\text { Pistia stratiotes } \\
\text { Eichornia azurea } \\
\text { Floscopa glabrata } \\
\text { Urochloa } \mathrm{spp}\end{array}$ & Urochloa spp. & $\begin{array}{c}\text { S. auriculata } \\
\text { S. biloba } \\
\text { P. stratiotes } \\
\text { E. azurea } \\
\text { F. glabrata } \\
\text { Typha domingensis } \\
\text { Urochloa } \text { spp. }\end{array}$ & Urochloa spp. & $\begin{array}{l}\text { S. auriculata } \\
\text { S. biloba } \\
\text { P. stratiotes } \\
\text { E. azurea } \\
\text { F. glabrata } \\
\text { Urochloa } \\
\text { spp. }\end{array}$ & Urochloa spp. \\
\hline River order & $4^{\mathrm{a}}$ & - & $3^{\mathrm{a}}$ & $3^{\mathrm{a}}$ & $3^{\mathrm{a}}$ & - & $2^{\mathrm{a}}$ \\
\hline $\begin{array}{l}\text { River width/ } \\
\text { Lake area }\end{array}$ & $\pm 24 \mathrm{~m}$ & $\pm 8.800 \mathrm{~m}^{2}$ & $\pm 8 \mathrm{~m}$ & $\pm 95 \mathrm{~m}$ & $\pm 25 \mathrm{~m}$ & $\pm 490 \mathrm{~m}^{2}$ & $\pm 7 \mathrm{~m}$ \\
\hline $\begin{array}{l}\text { Margin } \\
\text { declivity }\end{array}$ & low & low & low & low & low & low & low \\
\hline $\begin{array}{l}\text { Riparian } \\
\text { vegetation }\end{array}$ & $\begin{array}{l}\text { partially } \\
\text { preserved }\end{array}$ & partially preserved & $\begin{array}{l}\text { partially } \\
\text { preserved }\end{array}$ & partially preserved & $\begin{array}{l}\text { partially } \\
\text { preserved }\end{array}$ & $\begin{array}{l}\text { partially } \\
\text { preserved }\end{array}$ & $\begin{array}{l}\text { right margin - } \\
\text { preserved } \\
\text { left margin - } \\
\text { absent }\end{array}$ \\
\hline Substrate & mud and sand & $\begin{array}{l}\text { muddy and } \\
\text { decomposing } \\
\text { organic matter }\end{array}$ & mud and sand & $\begin{array}{l}\text { muddy and } \\
\text { decomposing } \\
\text { organic matter }\end{array}$ & $\begin{array}{l}\text { mud and } \\
\text { sand }\end{array}$ & $\begin{array}{l}\text { muddy and } \\
\text { decomposing } \\
\text { organic } \\
\text { matter }\end{array}$ & muddy \\
\hline
\end{tabular}

The highest fish diversity ( $\left.\mathrm{H}^{\prime} \geq 2.7, \mathrm{P} \leq 0.01\right)$ was observed in the Jacaré-Guaçu River down- and upstream the dam (S01 and S05), and in the Boa Esperança River (S03), the lowest values occurring in the reservoir and floodplain lakes. The diversity of fishes was positively correlated with depth $(\mathrm{r}=0.86, \mathrm{P}=0.01)$ and negatively correlated with water conductivity $(\mathrm{r}=-0.86, \mathrm{P}=0.01)$ (Table 4$)$. Thus, in the Jacaré-Guaçu basin, habitats with greater depth and lower values of conductivity tend to harbor a higher diversity of fish.

\section{Discussion}

The autochthonous fish species of Jacaré-Guaçu River represent $27 \%$ of all native species of the Upper Paraná River basin (Langeani et al. 2007). Taking into account the representativeness of the Jacaré-Guaçu River basin regarding the total area and diversity of habitats of the Upper Paraná River basin, this fish species richness is high. Compared to previous studies on Jacaré-Guaçu River basin (Albino 1987, Shibatta \& Silva-Souza 2008), 14 native species (Table 5) were not found during this study. The majority of these species was considered accidental species, and was collected downstream of the sampled area of this study, justifying the difficulty in catching them. Another possibility is that the populations of these species might be reduced, becoming restrict to specific habitats, or even locally extinct, as may be the case of Myloplus tiete, an endangered species of the Upper Paraná Rive Basin (Instituto... 2008).

The greater importance of Characiformes and Siluriformes, which represented $86 \%$ of all sampled species in this study, was reported by Langeani et al. (2007) in the fish fauna revision of the Upper Paraná River basin. These authors reported that $80 \%$ of all species belonged to the orders Characiformes and Siluriformes, most of the 
Table 2. Total number of collected specimens (N), constancy (co - constant, ac - accessory, ad - accidental, and ab - absent), and dominance of each species in the seven studied habitats (S; Figure 1). Specimens' abundance (\%; 0 means values less than 1$)$ of each species in the total sample, and origin of each of them. The migratory species (Carolsfeld et al. 2003) are highlighted in bold.

\begin{tabular}{lllllllll} 
Species & \multicolumn{9}{c}{ Constancy - Dominance } & & N & $\%$ & Origin $^{1}$ \\
\cline { 2 - 7 } & S01 & S02 & S03 & S04 & S05 & S06 & S07 &
\end{tabular}

Order Characiformes

Family Acestrorhynchidae

Acestrorhynchus lacustris (Lütken, 1875)

Family Anostomidae

Leporellus vitattus (Valenciennes, 1850)

Leporinus amblyrhynchus Garavello \& Britski, 1987

Leporinus friderici (Bloch, 1794)

Leporinus lacustris Campos, 1945

Leporinus octofasciatus Steindachner, 1915

Leporinus paranensis Garavello \& Britski, 1987

Leporinus striatus Kner, 1858

Schizodon borelli (Boulenger, 1900)

Schizodon nasutus Kner, 1858

Schizodon sp. 1

Family Characidae

Aphyocharax sp.

Astyanax altiparanae Garutti \& Britski, 2000

Astyanax fasciatus (Cuvier, 1819)

Astyanax paranae Eigenmann, 1914

Astyanax schubarti Britski, 1964

Bryconamericus stramineus Eigenmann, 1908

Gymnocorymbus ternetzi (Boulenger, 1895)

Hyphessobrycon bifasciatus (Ellis, 1911)

Hyphessobrycon eques (Steindachner, 1882)

Knodus moenkhausii (Eigenmann \& Kennedy, 1903)

Metynnis cf. mola Eigenmann \&Kennedy, 1903

Moenkhausia intermedia Eigenmann, 1908

Moenkhausia sanctafilomenae (Steindachner, 1907)

Oligosarcus pintoi Campos, 1945

Piabina argentea Reinhardt, 1867

Roeboides descalvadensis Fowler, 1932

Salminus hilarii Valenciennes, 1850

Serrapinnus cf. heterodon (Eigenmann, 1915)

Serrapinnus notomelas (Eigenmann, 1915)

Serrasalmus maculatus Kner, 1858

Serrasalmus marginatus Valenciennes, 1836

Triportheus nematurus (Kner, 1858)

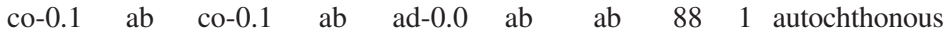

ad-0.0 ab $\quad$ ab $\quad$ ab $\quad$ ab $\quad$ ab $\quad$ ab $\quad 2 \quad 0$ autochthonous

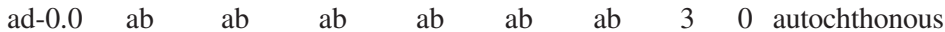
co-0.1 ac-0.0 co-0.0 ad-0.0 co-0.0 ad-0.1 ad-0.0 1032 autochthonous ac-0.0 ab $\quad a c-0.0 \quad a b \quad a b \quad a b \quad a b \quad 18 \quad 0$ autochthonous ad-0.0 ab $\quad a b \quad a b \quad a b \quad a b \quad a b \quad 3 \quad 0$ autochthonous ac-0.0 ab ad-0.0 ab $\quad$ ab $\quad$ ab $\quad$ ab $\quad 10 \quad 0$ autochthonous ac-0.0 ab ad-0.0 ad-0.0 ab $\quad$ ab $\quad$ ab $\quad 12 \quad 0$ autochthonous ad-0.0 ab $\quad \mathrm{ab} \quad \mathrm{ab} \quad \mathrm{ab} \quad \mathrm{ab} \quad \mathrm{ab} \quad 1 \quad 0$ autochthonous ad-0.0 ab ad-0.0 ad-0.0 ab ab ab $14 \quad 0$ autochthonous $\begin{array}{llllllllll}\mathrm{ad}-0.0 & \mathrm{ab} & \mathrm{ab} & \mathrm{ab} & \mathrm{ab} & \mathrm{ab} & \mathrm{ab} & 1 & 0\end{array}$

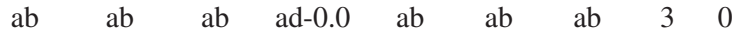

co-1.2 co-42.9 co-0.5 co-1.6 co-3.1 co-6.9 co-4.9 140421 autochthonous co-1.1 co-2.7 co-0.7 co-3.2 co-4.9 ac-0.6 ad-0.2 89913 autochthonous ac-0.1 ab $\quad$ ab $\quad a b \quad a b \quad a b \quad a b \quad 64 \quad 1$ autochthonous co-0.0 ad-0.0 ac-0.0 ab ab ab ab $34 \quad 0$ autochthonous ad-0.0 ab ad-0.0 ad-0.0 ac-0.0 ac-0.0 ad-0.7 $66 \quad 1$ autochthonous

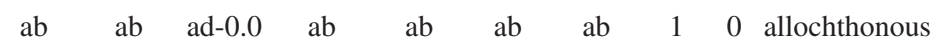
ab $\quad$ ab $\quad$ ab $\quad$ ad- $-0.0 \quad$ ab $\quad$ ad- $-0.0 \quad$ ab $\quad 14 \quad 0$ autochthonous ac-0.0 ad-0.0 ac-0.0 co-10.9 ac-0.0 ad-0.0 ac-0.1 5498 autochthonous

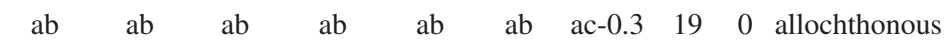
ad-0.0 ab $\quad \mathrm{ab} \quad \mathrm{ab} \quad \mathrm{ab} \quad \mathrm{ab} \quad \mathrm{ab} \quad \begin{array}{llll}7 & 0 & \text { allochthonous }\end{array}$ co-0.3 ad-0.0 co-0.3 ab ab ab ab $151 \quad 2$ autochthonous ab $\quad$ ab $\quad$ ad-0.0 ab $\quad$ ad-0.0 $\quad$ ab $\quad$ ad-0.2 $25 \quad 0$ autochthonous ac-0.0 ab ab co-0.0 ad- 0.0 ab ab $\quad 39 \quad 1$ autochthonous ac-0.1 ab ad-0.0 ad-0.0 ad-0.0 ad-0.0 ab $51 \quad 1$ autochthonous

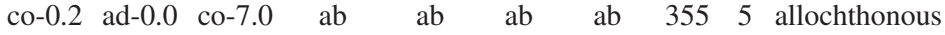
co-0.1 ac-0.0 ac-0.0 ad-0.0 ac-0.0 ad-0.1 ac-0.1 1112 autochthonous ab ad-0.0 ad-0.0 ab $\quad$ ab ab ab $32 \quad 0$ autochthonous ad-0.0 ad-0.0 ac-0.0 co-0.6 co-0.3 co-5.3 ab 3395 autochthonous

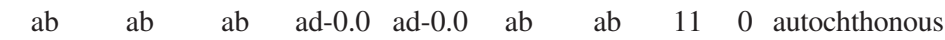
ad-0.0 ab ad-0.0 $\quad$ ab $\quad$ ab $\quad$ ab $\quad$ ab $\quad 9 \quad 0$ allochthonous ad-0.0 ab $\quad \mathrm{ab} \quad \mathrm{ab} \quad \mathrm{ab} \quad \mathrm{ab} \quad \mathrm{ab} \quad 6 \quad 0$ allochthonous

${ }^{1}$ Buckup et al. (2007), Júlio Jr. et al. (2009) and Langeani et al. (2007). 
Table 2. Continued...

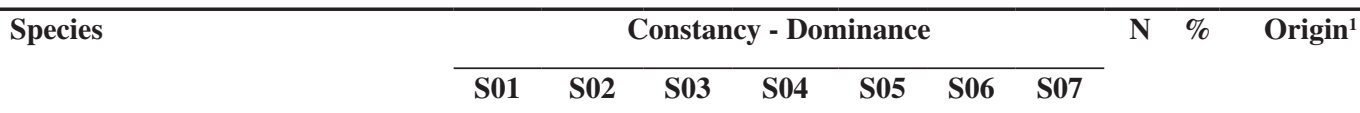

Family Crenuchidae

Characidium gomesi Travassos, 1956

Characidium zebra Eigenmann, 1909

Family Curimatidae

Cyphocharax modestus (Fernández-Yépez, 1948)

Cyphocharax nagelii (Steindachner, 1881)

Steindachnerina insculpta (Fernández-Yépez, 1948)

Family Erythrinidae

Hoplerythrinus unitaeniatus (Agassiz, 1829)

Hoplias malabaricus (Bloch, 1794)

Family Lebiasinidae

Pyrrhulina australis Eigenmann \& Kennedy, 1903

Family Parodontidae

Apareiodon affinis (Steindachner, 1879)

Parodon nasus Kner, 1859

Family Prochilodontidae

Prochilodus lineatus (Valenciennes, 1836)

Order Cyprinodontiformes

Family Poeciliidae

Poecilia reticulata Peters, 1859

Phalloceros caudimaculatus (Hensel, 1868)

Order Gymnotiformes

Family Gymnotidae

Gymnotus aff. carapo Linnaeus, 1758

Family Sternopygidae

Eigenmannia trilineata López \& Castello, 1966

Sternophygus macrurus (Bloch \& Schneider, 1801)

Order Perciformes

Family Cichlidae

Geophagus brasiliensis (Quoy \& Gaimard, 1824)

Cichla kelberi Kullander \& Ferreira, 2006

Cichlasoma paranaense Kullander, 1983

Crenicichla britski Kullander, 1982

Family Scianidae

Plagioscion squamosissimus (Heckel, 1840)

Order Siluriformes

Family Auchenipteridae

Tatia neivai (Von Ihering, 1930)

Trachelyopterus coriaceus Valenciennes, 1840

$\begin{array}{cccccccccc}\text { ad-0.0 } & \text { ad-0.0 } & \text { ab } & \text { ab } & \text { ab } & \text { ab } & \text { ab } & 2 & 0 & \text { autochthonous } \\ \text { ac-0.0 } & \text { ab } & \text { ad-0.0 } & \text { ab } & \text { ac-0.0 } & \text { co-0.1 } & \text { ac-0.0 } & 33 & 0 & \text { autochthonous } \\ & & & & & & & & & \\ \text { ac-0.0 } & \text { ab } & \text { ac-0.0 } & \text { ac-0.0 } & \text { ad-0.0 } & \text { ad-0.0 } & \text { ab } & 39 & 1 & \text { autochthonous } \\ \text { ab } & \text { ab } & \text { ad-0.0 } & \text { ab } & \text { ad-0.0 } & \text { ab } & \text { ab } & 4 & 0 & \text { autochthonous } \\ \text { co-9.1 } & \text { co-0.2 } & \text { co-4.4 } & \text { co-1.7 } & \text { co-1.3 } & \text { co-0.2 } & \text { ab } & 1073 & 16 & \text { autochthonous }\end{array}$

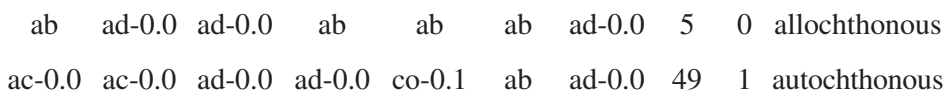

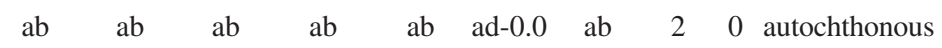

co-0.0 ab ac-0.0 ab ad- $0.0 \quad$ ab $\quad$ ab $\quad 32 \quad 0$ autochthonous

$\begin{array}{lllllllll}\mathrm{ad}-0.0 & \mathrm{ab} & \mathrm{ab} & \mathrm{ab} & \mathrm{ab} & \mathrm{ab} & \mathrm{ab} & 2 & 0\end{array}$

co-0.3 ac-0.1 ac-0.0 co-0.0 co-0.2 ad-0.1 co-0.7 $223 \quad 3$ autochthonous

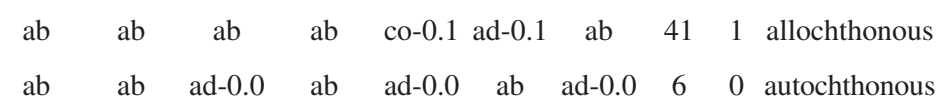

co-0.0 ac-0.0 co-0.1 co-0.0 co-0.2 co-0.1 co-1.2 $150 \quad 2$ autochthonous

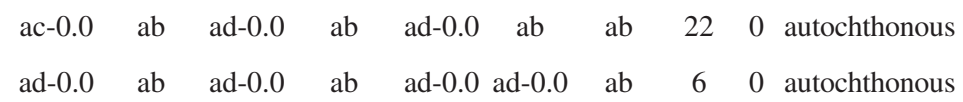

ad-0.0 ad-0.0 ad-0.0 ac-0.0 ad-0.0 ad-0.2 ad-0.0 $49 \quad 1$ autochthonous

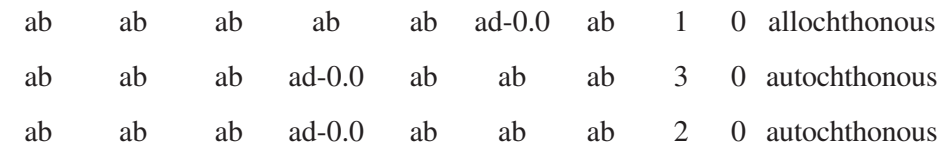

ac-0.0 ab $\quad$ ab $\quad a b \quad a b \quad a b \quad a b \quad 32 \quad 0$ allochthonous

${ }^{1}$ Buckup et al. (2007), Júlio Jr. et al. (2009) and Langeani et al. (2007). 
Table 2. Continued...

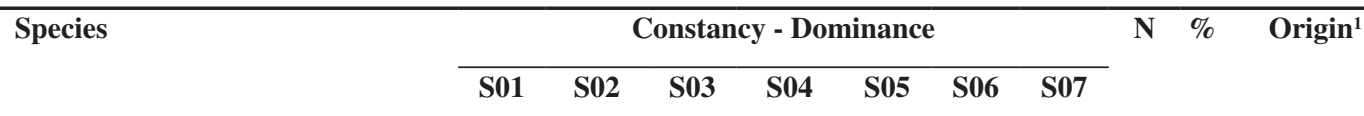

\begin{tabular}{|c|c|c|c|c|c|c|c|}
\hline amily Callichthyidae & & & & & & & \\
\hline Corydoras aeneus (Gill, 1858) & $a b$ & ad- 0.0 & $a b$ & $a b$ & ad- 0.0 & 10 & 0 autochthonous \\
\hline Hoplosternum littorale (Hancock, 1828) & ac- -0.0 ad- 0.0 & ad- 0.0 & co- 0.2 & co- 0.3 ad- -0.0 & ac- 0.4 & 143 & 2 autochthonous \\
\hline Lepthoplosternum pectorale (Boulenger, 1895) & ac- 0.0 ad- 0.0 & ac- -0.0 & ac- -0.0 & ad- 0.0 co- 0.3 & ac- 0.4 & 119 & 2 autochthonous \\
\hline
\end{tabular}

Family Cetopsidae

Cetopsis gobioides Kner, 1857

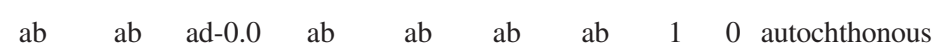

Family Clariidae

Clarias gariepinus (Burchell, 1822)

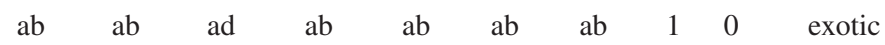

Family Doradidae

Rhinodoras dorbignyi (Kner, 1855)

Family Heptapteridae

Imparfinis schubarti (Gomes, 1956)

ad-0.0 ab $\quad \mathrm{ab} \quad \mathrm{ab} \quad \mathrm{ab} \quad \mathrm{ab} \quad \mathrm{ab} \quad 5 \quad 0$ autochthonous

Pimelodella aff. gracilis (Valenciennes in d'Orbigny, 1835) ad-0.0 ab ad-0.0 ab ad-0.0 ad-0.0 ad-0.0 $20 \quad 0$ autochthonous

Rhamdia quelen (Quoy \& Gaimard in Freycinet, 1824) $\quad$ ab $\quad$ ab $\quad$ ab $\quad$ ad- 0.0 ad- $0.0 \quad$ ab $\quad$ ab $\quad 9 \quad 0$ autochthonous Family Loricariidae

Hypostomus albopunctatus (Regan, 1908)

Hypostomus ancistroides (Ihering, 1911)

ad-0.0 ab ad-0.0 ab $\quad$ ab $\quad$ ab $\quad$ ab $\quad 8 \quad 0$ autochthonous

Hypostomus margaritifer (Regan, 1908)

co-0.0 ad-0.0 ac-0.0 ad-0.0 ac-0.0 ad-0.0 ac-0.4 $74 \quad 1$ autochthonous

Hypostomus nigromaculatus (Schubart, 1964)

ad-0.0 ab $\quad$ ab $\quad$ ad- $0.0 \quad$ ab $\quad$ ab $\quad$ ab $\quad 9 \quad 0$ autochthonous

Hypostomus regani (Ihering, 1905)

ad-0.0 ab $\quad$ ab $\quad$ ab $\quad$ ad- $-0.0 \quad$ ab $\quad$ ab $\quad 9 \quad 0$ autochthonous

Hypostomus sp. 1

ad-0.0 ad-0.0 ab $\quad \mathrm{ab} \quad \mathrm{ab} \quad \mathrm{ab} \quad \mathrm{ab} \quad 8 \quad 0$ autochthonous

Hypostomus sp. 2

ad-0.0 ad-0.0 ad-0.0 ab ab ad-0.0 ac-0.1 $19 \quad 0$

Hypostomus sp. 3

ac- $-0.0 \quad$ ab $\quad$ ad- $0.0 \quad$ ab $\quad$ ab $\quad$ ab $\quad$ ab $\quad 9 \quad 0$

Liposarcus anisitsi (Eigenmann \& Kennedy, 1903)

ad-0.0 ab ab ab $\quad$ ab $\quad$ ab $\quad$ ab $\quad 3 \quad 0$

Hisonotus sp.

ad-0.0 ab $\quad$ ab $\quad$ ad-0.0 $\quad$ ad-0.0 $\quad$ ab $\quad$ ad-0.0 $14 \quad 0$ allochthonous

Loricaria prolixa Isbrücker \& Nijssen, 1978

ad-0.0 ad-0.0 ad-0.0 ad-0.0 ab ad-0.2 ad-0.0 $58 \quad 1$

Otothyropsis marapoama Ribeiro, Carvalho \& Melo, 2005 ad-0.0 $\quad$ ab $\quad$ ac- $-0.1 \quad$ ab $\quad$ ab $\quad$ ab $\quad$ ab $\quad 32 \quad 0$ autochthonous

Pseudotocinclus sp.

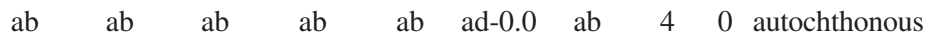

Family Pseudopimelodidae

Microglanis garavelloi Shibatta \& Benine, 2005

Pseudopimelodus mangurus (Valenciennes, 1840)

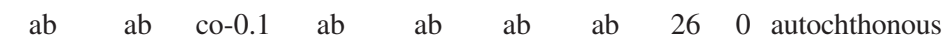

ad-0.0 ab ad-0.0 ab $\quad$ ab $\quad$ ab $\quad$ ab $\quad 8 \quad 0$ autochthonous

Family Trichomycteridae

Paravandellia oxyptera Miranda Ribeiro, 1912

ab $\quad$ ab $\quad$ ad- $-0.0 \quad$ ab $\quad$ ab $\quad$ ab $\quad$ ab $\quad 1 \quad 0$ autochthonous

Order Synbranchiformes

Family Synbranchidae

Synbranchus marmoratus Bloch, 1785

ad-0.0 ad-0.0 ad-0.0 ad-0.0 ac-0.0 ad-0.0 ab $15 \quad 0$ autochthonous

'Buckup et al. (2007), Júlio Jr. et al. (2009) and Langeani et al. (2007). 
Table 3. Shannon-Wiener (H') index, Jaccard index (\%; lower matrix) and Student's $t$-test (P; upper matrix) of H' differences between the studied sites (S; Figure 1).

\begin{tabular}{lcccllll}
\hline & S01 & S02 & S03 & S04 & S05 & S06 & S07 \\
\hline H' & 2.8 & 1.4 & 2.7 & 2.1 & 2.6 & 2.4 & 2.5 \\
S01 & - & 0.00 & 0.44 & 0.00 & 0.00 & 0.00 & 0.00 \\
S02 & 36 & - & 0.00 & 0.00 & 0.00 & 0.00 & 0.00 \\
S03 & 57 & 42 & - & 0.00 & 0.01 & 0.00 & 0.01 \\
S04 & 37 & 42 & 35 & - & 0.00 & 0.00 & 0.00 \\
S05 & 42 & 36 & 47 & 54 & - & 0.00 & 0.11 \\
S06 & 34 & 46 & 39 & 50 & 54 & - & 0.01 \\
S07 & 28 & 33 & 40 & 39 & 47 & 47 & - \\
\hline
\end{tabular}

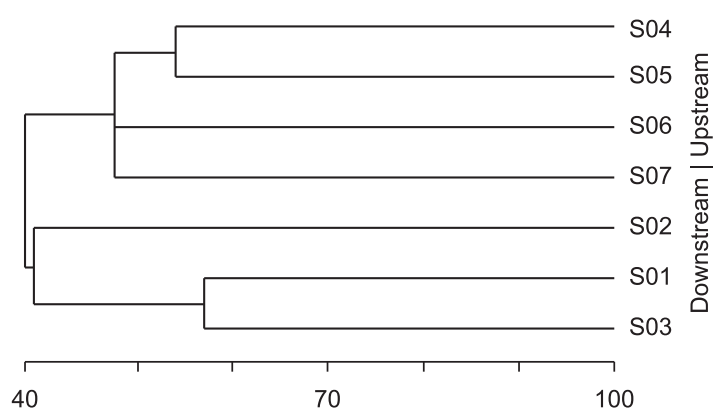

Figure 2. Dendrogram of fish species similarity (Jaccard index) among the studied sites (S; Figure 1) up- and downstream the Gavião Peixoto Dam.

Table 4. Pearson's Correlation between the Shannon-Wiener index and the physical and chemical variables of the studied sites (Figure 1).

\begin{tabular}{lcc}
\hline \multicolumn{1}{c}{ Variables } & r & P \\
\hline Depth $(\mathrm{m})$ & 0.86 & 0.01 \\
Current velocity $(\mathrm{m} / \mathrm{s})$ & 0.73 & 0.07 \\
Vertical transparency $(\mathrm{m})$ & 0.06 & 0.89 \\
Water temperature $\left({ }^{\circ} \mathrm{C}\right)$ & -0.31 & 0.49 \\
$\mathrm{pH}$ & 0.68 & 0.09 \\
Dissolved oxygen $\left(\mathrm{mg} . \mathrm{L}^{-1}\right)$ & 0.51 & 0.25 \\
Water conductivity $\left(\mu \mathrm{S} . \mathrm{cm}^{-1}\right)$ & -0.86 & 0.01 \\
\hline
\end{tabular}

dominant groups of this basin also belonging to these two orders. The predominance of these orders has been reported in several studies on tributaries of the Upper Paraná River basin (Uieda 1984, Garutti 1988, Penczak et al. 1994, Castro \& Casatti 1997, Pavanelli \& Caramaschi 1997, Casatti et al. 2001, Castro et al. 2003, 2004, 2005, Langeani et al. 2005, Perez-Jr. \& Garavello 2007, Apone et al. 2008, Meschiatti \& Arcifa 2009), where similar contributions of the orders to those observed in this study have been found. Moreover, this pattern of representativeness of the orders was reported in non-estuarine streams of the Neotropical region, Amazon streams, São Francisco River basin streams, Atlantic Forest streams, and in the Eastern Chaco of Argentina (reviewed by Castro et al. 2003). All these studies highlight the diversity and adaptive success of these two orders of Otophysi in the Neotropical region, especially in South America (Reis et al. 2003, Briggs 2005, Buckup et al. 2007, Ferraris 2007).

Most species belong to the order Characiformes, which contributed $86.3 \%$ to the overall captured specimens. Characidae is the most abundant family, particularly the species Astyanax altiparanae.
The dominance of this species has been reported in several habitats of the Upper Paraná River basin (Uieda 1984, Garutti 1988, Penczak et al. 1994, Castro \& Casatti 1997, Pavanelli \& Caramaschi 1997, Castro et al. 2003, 2004, 2005). Several reasons may be raised to explain the dominance of A. altiparanae, Castro et al. (2004) mentioning three in particular: to belong to the omnivorousq guild, with a high trophic amplitude, for being a nektonic species and an agile swimmer that is distributed from the middle to the surface of the water (Casatti et al. 2001), and to present a high reproductive plasticity (Godoy 1975, Garutti 1989, Agostinho \& Julio Jr. 1999).

The high occurrence of accidental species can be justified by several reasons (Garutti 1988, Oliveira \& Garavello 2003), such as: physical and chemical seasonal variations of the habitats, which could cause alterations in the population size of a species, influencing the capture probability; movement of the shoals, which can facilitate or hinder the capture of individuals of a species; and sampling methodology, since the sample devices are selective for species, due mainly to the wide behavioral range of tropical fish species. Besides, the size and situation of the fish populations, along the time, can direct influence its constancy.

Probably, the heterogeneity among the studied habitats ensured the establishment of a particular fish fauna, one of the reasons for the low similarity observed. This heterogeneity can also be responsible for a theoretical influence on the efficiency of the methods, which could result in a differential selectivity of the number of specimens and of species collected in each habitat, ending in the low fish fauna similarity. The greater similarity between the Jacaré-Guaçu River, downstream the dam, and its tributary Boa Esperança River is probably due to the fact they are close and, consequently, share the fish fauna. The low similarity among habitats down- and upstream the dam indicates the existence of a particular fish fauna in these two regions, certainly ensured by the loss of connectivity, almost a century ago.

Since 1913, when the dam was closed, at least four fish ladders have been built on the Gavião Peixoto Dam (see Esguicero \& Arcifa $2010 \mathrm{~b}$ for more details). The connection between up- and downstream areas has been intermittent, and in addition, the efficiency of the last two fish ladders for reproductive migration of migratory fish species was very low. Due to the morphological characteristics of the river channel, of Gavião Peixoto Dam, and of the fish ladders, these facilities presented problems related to flow control (Esguicero $\&$ Arcifa 2010b), making them unattractive for the reproductive migration of migratory fish species.

Based on the low fish fauna similarity between the areas down- and upstream the dam, and all the problems for fish dispersion caused by the Gavião Peixoto Dam, we can hypothesize that both reproductive migration and migration for dispersion were negatively influenced, affecting, therefore, both migratory and non-migratory fish species. An example of this fact is that the river damming and all the history of fish passages have probably difficulted the dispersion of the majority of the alien species found downstream the dam. Of the 13 alien species captured, only five were sampled upstream the dam. In addition to the possibility of dispersion through the fish ladders, accidental or deliberate release by local people could be other ways these five species have colonized upstream habitats. According to Langeani et al. (2007), the fish farming, sportfishing, fishkeeping, and biological control are important sources of alien fish species in the Upper Paraná River Basin.

Most allochthonous species, caught in this study, invaded the Upper Paraná River basin after the waterfall Salto de Sete Quedas was submerged by the Itaipu Reservoir (Langeani et al. 2007, Júlio Jr. et al. 2009). The invasion and adaptive success of alien species are a major threat to the worldwide fish fauna (Rodríguez 2001, Pelicice \& Agostinho 2009). In general, alien species show a rapid 
Table 5. Species reported in previous studies on Jacaré-Guaçu River basin (Albino 1987, Shibatta \& Silva-Souza 2008) that were not found during this study. RF - Feijão River basin; downstream - species captured downstream from the studied region.

\begin{tabular}{|c|c|c|}
\hline Species & Constancy & Sample site \\
\hline \multicolumn{3}{|l|}{ Order Characiformes } \\
\hline \multicolumn{3}{|l|}{ Family Anostomidae } \\
\hline Leporinus obtusidens Valenciennes, 1836 & accidental & downstream \\
\hline \multicolumn{3}{|l|}{ Family Characidae } \\
\hline Bryconamericus iheringii (Boulenger, 1887) & - & $\mathrm{RF}$ \\
\hline Hemigrammus marginatus Ellis, 1911 & accidental & downstream \\
\hline Hyphessobrycon anisitsi (Eigenmann, 1907) & - & \\
\hline Myloplus tiete (Eigenmann \& Norris, 1900) & accidental & downstream \\
\hline \multicolumn{3}{|l|}{ Family Parodontidae } \\
\hline Apareiodon ibitiensis Campos, 1944 & accidental & downstream \\
\hline Apareiodon piracicabae (Eigenmann, 1907) & - & $\mathrm{RF}$ \\
\hline \multicolumn{3}{|l|}{ Order Siluriformes } \\
\hline \multicolumn{3}{|l|}{ Family Callichthyidae } \\
\hline Callichthys callichthys (Linnaeus, 1758) & accidental & downstream \\
\hline \multicolumn{3}{|l|}{ Family Heptapteridae } \\
\hline Cetopsorhamdia iheringi Schubart \& Gomes, 1959 & accidental & downstream \\
\hline \multicolumn{3}{|l|}{ Family Pimelodidae } \\
\hline Hemisorubim platyrhynchos (Valenciennes, 1840) & accidental & downstream \\
\hline Iheringichthys labrosus (Lütken, 1874) & accessory & downstream \\
\hline Pimelodus maculatus La Cepède, 1803 & constant & downstream \\
\hline \multicolumn{3}{|l|}{ Family Loricariidae } \\
\hline Corumbataia cuestae Britski, 1997 & - & $\mathrm{RF}$ \\
\hline Rineloricaria sp. & accidental & downstream \\
\hline \multicolumn{3}{|l|}{ Family Trichomycteridae } \\
\hline Trichomycterus sp. & - & $\mathrm{RF}$ \\
\hline
\end{tabular}

colonization process, probably due to better adaptation to ecological conditions in relation to the native fish fauna (Rodríguez 2001). An example of the impact caused by the introduction of alien species is the absence of Serrasalmus maculatus, the only native representative of the genera Serrasalmus in the Upper Paraná River basin, in samples caught downstream the Gavião Peixoto dam. According to Agostinho \& Julio Jr. (2002), this species was being excluded from the Paraná River, in the region of Itaipu reservoir, by the introduction of S. marginatus, after the disappearance of Salto de Sete Quedas. These authors mention that in the Middle Paraná River, near Yaciretá Dam, S. maculatus and S. marginatus occur sympatrically, in a proportion of 1:4.5. Therefore, the absence of captures of the species $S$. maculatus downstream the Gavião Peixoto dam is probably a result of the drastic local reduction, or even extinction, of its population by the invasion of $S$. marginatus. This species was captured only downstream the dam, and the drawback for its upstream dispersion can be one of the few reasons for the persistence of $S$. maculatus population upstream the dam. A similar case was reported in the Piquiri River (Agostinho \& Julio Junior 2002), where the abundance of $S$. maculatus remained high due to the presence of Nhá Bárbara and Apertado Falls, which prevented the dispersion of $S$. marginatus to upstream areas.

There is a clear difference between the fish fauna composition of habitats down- and upstream the dam, indicated by higher values of H' observed in the habitats downstream the dam, and a higher number of fish species captured in these habitats. According to some authors (Peres Neto et al. 1995, Uieda \& Barreto 1999, Súarez 2008), several ecological factors, such as food availability and habitat structure, can be responsible for the fish fauna composition and diversity. The existence of a higher floodplain area downstream the Gavião Peixoto Dam (Esguícero \& Arcifa 2010b), and the free section downstream the Jacaré-Guaçu River confluence with the Tietê River (downstream the Ibitinga Dam), could support a higher number of fish species 
in that stretch. Another factor is that depth of the water body can positively influence the population size and the number of fish species (Súarez 2008), what was also observed in this study. Eutrophication and pollution affect negatively the fish fauna, what can be indicated by the negative correlation of fish diversity and water conductivity (Larkin \& Northcote 1969, Bauer 1986), as observed in this study.

Carolsfeld et al. (2003) report the existence of ca. 16 migratory fish species, medium to large-sized Characiformes and Siluriformes, in the Upper Paraná River basin. This number is much higher than that found in the Jacaré-Guaçu River, where only 4 medium-sized migratory species of Characiformes were caught. Currently, there is an intense loss of fish diversity in the Upper Paraná River basin, the main causes being the local extinction of several migratory species, the overfishing, the pollution, and the loss of nursery habitats (Agostinho et al. 2007). All these impact sources are common in the Tietê River, which has a succession of reservoirs, drastically decreasing the fish richness, mainly of the migratory ones.

\section{Acknowledgements}

We thank all the people who have helped in the field work, CPFL Energy for logistical support, RMC Castro and the Laboratório de Ictiologia de Ribeirão Preto, Universidade de São Paulo, Ribeirão Preto, and CAPES (Coordenação de Aperfeiçoamento de Pessoal de Nível Superior) for a grant to ALHE. We also thank the Programa de Pós-Graduação em Biologia Comparada da Universidade de São Paulo for financial support and S.M. Thomaz, R. Fuji, and two anonymous referees for valuable suggestions on the manuscript.

\section{References}

AGOSTINHO, A.A. \& JULIO Jr., H.F. 1999. Peixes da Bacia do Alto Rio Paraná. In Estudos ecológicos de comunidades de peixes tropicais (R.H. Lowe-McConnell). Edusp, São Paulo, p.374-400.

AGOSTINHO, C.S. \& JULIO Jr., H.F. 2002. Observation of an invasion of the piranha Serrasalmus marginatus Velenciennes, 1947 (Osteichthyes, Serrasalmidae) into the Upper Parana River, Brazil. Acta Sci. 24(2):391-395.

AGostinhO, A.A., GOMES, L.C. \& PELICICE, F.M. 2007. Ecologia e manejo de recursos pesqueiros em reservatórios do Brasil. EDUEM, Maringá.

ALBINO, A.L.D. 1987. Estudo sobre a fauna de peixes da bacia do rio JacaréGuaçu (Estado de São Paulo) com uma avaliação preliminar dos efeitos de dois barramentos. Dissertação de Mestrado, Universidade Federal de São Carlos, São Carlos.

APONE, F., OLIVEIRA, A.K. \& GARAVELLO, J.C. 2008. Ichthyofaunistic composition of the Quilombo river, tributary of the Mogi-Guaçu River, Upper Paraná River basin, southeastern Brazil. Biota Neotrop. 8(1): http:// www.biotaneotropica.org.br/v8n1/pt/abstract?article+bn02208012008.

BAUER, G. 1986. The status of the freshwater pearl mussel Margaritifera margaritifera L. in the south of its European range. Biol. Conserv. 38(1):1-9.

BRIGGS, J.C. 2005. The biogeography of otophysan fishes (Ostariophysi: Otophysi): a new appraisal. J. Biogeogr. 32(2):287-294.

BROWER, J.E. \& ZAR, J.H. 1984. Field and laboratory methods for general ecology. Wm.C. Brown Publishers, Dubuque, Iowa.

BUCKUP, P.A., MENEZES, N.A. \& GHAZZI, M.S.A. 2007. Catálogo das Espécies de peixes de água doce do Brasil. Museu Nacional, Rio de Janeiro.

CAROLSFELD, J., HARVEY, B., ROSS, C. \& BAER, A. 2003. Migratory Fishes of South America: Biology, Fisheries, and Conservation Status. World Fisheries Trust; World Bank; IDRC, Ottawa.
CASATTI, L., LANGEANI, F. \& CASTRO, R.M.C. 2001. Peixes de riacho do Parque Estadual Morro do Diabo, bacia do alto rio Paraná, SP. Biota Neotrop. 1(1): http://www.biotaneotropica.org.br/v1n12/ pt/abstract?in ventory+BN00201122001.

CASTRO, R.M.C. \& CASATTI, L. 1997. The fish fauna from a small forest stream of the upper Paraná River basin, southeastern Brazil. Ichthyol. Explor. Fresh. 7(3):337-352.

CASTRO, R.M.C., CASATTI, L., SANTOS, H.F., FERREIRA, K.M., RIBEIRO, A.C., BENINE, R.C., DARDIS, G.Z.P., MELO, A.L.A., STOPIGLIA, R., ABREU, T.X., BOCKMANN, F.A., CARVALHO, M., GIBRAN, F.Z. \& LIMA, F.C. 2003. Estrutura e composição da ictiofauna de riachos do rio Paranapanema, sudeste e sul do Brasil. Biota Neotrop. 3(1): http://www.biotaneotropica.org.br/v3n1/ pt/ abstract?article+BN01703012003.

CASTRO, R.M.C., CASATTI, L., SANTOS, H.F., MELO, A.L.A., MARTINS, L.S.F., FERREIRA, K.M., GIBRAN, F.Z., BENINE, R.C., CARVALHO, M. \& RIBEIRO, A.C., ABREU, T.X., BOCKMANN, F.A., PELIÇÃO, G.Z., STOPIGLIA, R. \& LANGEANI, F. 2004. Estrutura e composição da ictiofauna de riachos da bacia do rio Grande no estado de São Paulo, sudeste do Brasil. Biota Neotrop. 4(1): http://www.biotaneotropica.org. br/v4n1/pt/abstract?article+BN01704012004.

CASTRO, R.M.C., CASATTI, L., SANTOS, H.F., VARI, R.P., MELO, A.L.A., MARTINS, L.S.F., ABREU, T.X., BENINE, R.C., GIBRAN, F.Z., RIBEIRO, A.C., BOCKMANN, F.A., CARVALHO, M., PELIÇÃO, G.Z.P., FERREIRA, K.M., STOPIGLIA, R. \& AKAMA, A. 2005. Structure and composition of the stream ichthyofauna of four tributary rivers of the Upper Paraná River basin, Brazil. Ichthyol. Explor. Fresh. 16(3):193-214.

COMPANHIA DE TECNOLOGIA DE SANEAMENTO AMBIENTAL. 2010. http://www.cetesb.sp.gov.br. Accessed: August/2010.

CORBI, J.J., STRIXINO, S.T., SANTOS, A. \& DEL GRANDE, M. 2006. Diagnóstico ambiental de metais e organoclorados em córregos adjacentes a áreas de cultivo de cana-de-açúcar (Estado de São Paulo, Brasil). Quim. Nova 29(1):61-65.

ESGUÍCERO, A.L.H. \& ARCIFA, M.S. 2010a. Programa de monitoramento da ictiofauna - Fase de Operação (Julho/2007 a Fevereiro/2010). JGP Consultoria e Participações Ltda, São Paulo.

ESGUÍCERO, A.L.H. \& ARCIFA, M.S. 2010b. Fragmentation of a Neotropical migratory fish population by a century-old dam. Hydrobiologia 638(1):41-53.

FERRARIS, C.J. 2007. Checklist of catfishes, recent and fossil (Osteichthyes: Siluriformes), and catalogue of siluriform primary types. Zootaxa 1418(1):1-628.

GARUTTI, V. 1988. Distribuição longitudinal da ictiofauna em um córrego da região noroeste do estado de São Paulo, bacia do rio Paraná. Rev. Brasil. Biol. 48(4):747-759.

GARUTTI, V. 1989. Contribuição ao conhecimento reprodutivo de Astyanax bimaculatus (Ostariophysi, Characidae), em cursos de água da bacia do rio Paraná. Rev. Brasil. Biol. 49(2):489-495.

GÉRY, J. 1969. The freshwater fishes of South America. In Biogeography and Ecology in South-America (E.J. Fittkau, J. Illies, H. Klinge, G.H. Schwabe, \& H. Sioli, eds). Junk, The Hague, p.828-848.

GODOY, M.P. 1975. Peixes do Brasil, Subordem Characoidei: bacia do rio Mogi-Guaçu. Editora Franciscana, Piracicaba.

HAMMER, Ø., HARPER, D.A.T. \& RYAN, P.D., 2001. PAST: paleontological statistics software package for education and data analysis. 1.90.

HUECK, K. \& SEIBERT, P. 1981. Vegetationskarte von Südamerika. Folia Geobotanica 17(4):90.

INSTITUTO BRASILEIRO DE GEOGRAFIA E ESTATÍSTICA. 2000. http:// www.ibge.gov.br. Accessed: August/2010.

INSTITUTO BRASILEIRO DO MEIO AMBIENTE E DOS RECURSOS NATURAIS RENOVÁVEIS - IBAMA. 2008. http://www.mma.gov.br/ port/sbf/ fauna/ index.cfm (ultimo accesso em 01/2011). 
JULIO Jr., H.F., TÓS, C.D., AGOSTINHO, A.A. \& PAVANELLI, C.S. 2009 A massive invasion of fish species after eliminating a natural barrier in the upper rio Paraná basin. Neotrop. Ichthyol. 7(1):709-718.

LANGEANI, F. 1989. Ictiofauna do alto curso do rio Tietê (SP): taxonomia. Instituto de Biociências, Tese de Doutorado, Universidade de São Paulo.

LANGEANI, F., CASATTI, L., GAMEIRO, H.S., CARMO, A.B. \& ROSSAFERES, D.C. 2005. Riffle and pool fish communities in a large stream of southeastern Brazil. Neotrop. Ichthyol. 3(1) 305-311.

LANGEANI, F., CASTRO, R.M.C., OYAKAWA, O.T., SHIBATTA, O.A., PAVANELLI, C.S. \& CASATTI, L. 2007. Diversidade da ictiofauna do Alto Rio Paraná: composição atual e perspectivas futuras. Biota Neotrop. 7(3): http://www.biotaneotropica.org.br/v7n3/pt/ abstract?article+bn0340703 2007.

LARKIN, P.A. \& NORTHCOTE, T.G. 1969. Fish as Indices of Eutrophication. In Eutrophication: causes, consequences, correctives (P.A. Larkin, T.G. Northcote \& G.A. Rohlich, eds). National Academy of Sciences, Washington, p.256-273.

LEVANTAMENTO DE UNIDADES DE PRODUÇÃO AGROPECUÁRIA DO ESTADO DE SÃO PAULO. 2008. http://www.cati.sp.gov.br/ projetolupa. Accessed: August/2010

LUDWIG, J.A. \& REYNOLDS, J.F. 1988. Statistical ecology: a primer on methods and computing. Wiley-Interscience, New York.

MESCHIATTI, A.J. \& ARCIFA, M.S. 2009. A review on the fishfauna of Mogi-Guaçu River basin: a century of studies. Acta Limnol. Brasil. 21(1):135-159.

NIMER, N. 1989. Climatologia do Brasil. Instituto Brasileiro de Geografia e Estatística, Rio de Janeiro.

OLIVEIRA, A.K. \& GARAVELLO, J.C. 2003. Fish assemblage composition in a tributary of the Mogi Guaçu river basin, southeastern Brazil. Iheringia Série Zool. 93:127-138.

PAVANELLI, C.S. \& CARAMASCHI, E.P. 1997. Composition of the ichthyofauna of two small tributaries of the Paraná river, Porto Rico, Paraná State, Brazil. Ichthyol. Explor. Fresh. 8(2):23-32.
PELICICE, F.M. \& AGOSTINHO, A.A. 2009. Fish fauna destruction after the introduction of a non-native predator (Cichla kelberi) in a Neotropical reservoir. Biol. Invasions 11(8):1789-1801.

PENCZAK, T., AGOSTINHO, A.A. \& OKADA, E.K. 1994. Fish diversity and community structure in two small tributaries of the Paraná River, Paraná State, Brazil. Hydrobiologia 294(3):243-251.

PERES NETO, P.R., BIZERRIL, C., IGLESIAS, R. \& ESTEVES, F.A. 1995. An overview of some aspects of river ecology: a case study on fish assemblages distribution in an eastern Brazilian coastal river. Oecol. Brasil. 1(1):317-334.

PEREZ-JUNIOR, O.R. \& GARAVELLO, J.C. 2007. Ictiofauna do ribeirão do Pântano, afluente do rio Mogi-Guaçu, bacia do alto rio Paraná, São Paulo, Brasil. Iheringia Série Zool. 97(3):328-335.

REIS, R.E., KULLANDER, S.O. \& FERRARIS, C.J. 2003. Check List of the Freshwater Fishes of South and Central America. EDIPUCRS, Porto Alegre.

RODRÍGUEZ, J.P. 2001. Exotic species introductions into South America: an underestimated threat? Biodivers. Conserv. 10(11):1983-1996.

SHIBATTA, O.A. \& SILVA-SOUZA, A.T. 2008. Fish, Ribeirão do Feijão Basin, São Carlos, São Paulo, Brazil. Check List 4(1):75-78.

SÚAREZ, Y.R. 2008. Spatial and temporal variation in fish species diversity and composition in streams of Ivinhema River basin, upper Paraná River. Biota Neotrop. 8(3): http://www.biotaneotropica.org.br/v8n3/pt/ abstract?article+bn02308032008.

UIEDA, V.S. 1984. Ocorrência e distribuição dos peixes em um riacho de água doce. Rev. Brasil. Biol. 44(2):203-213.

UIEDA, V.S. \& BARRETTO, M.G. 1999. Composição da ictiofauna de quatro trechos de diferentes ordens do rio Capivari, bacia do Tietê, Botucatu, São Paulo. Rev. Brasil. Biol. 18(4):413-416.

VARI, R.P. 1988. The Curimatidae, a lowland neotropical fish family (Pisces: Characiformes); distribution, endemism, and phylogenetic biogeography. In Proceedings of a Workshop on Neotropical Distribution Patterns (W.R. Heyer, \& P.E. Vanzolini, eds). Academia Brasileira de Ciências, Rio de Janeiro, p.343-377. 